\section{Adding group psychotherapy to routine care does not improve outcomes in adolescents who repeatedly self-harm}

\section{QUESTION}

Question: Is group therapy more effective than routine care at decreasing the frequency of self-harm episodes in adolescents who repeatedly self-harm, and is it cost-effective?

Patients: Adolescents aged between 12 and 17 years who repeatedly self-harmed ( $\geq 2$ episodes within the year before the study). Non-English speakers, those with severe anorexia nervosa, a current psychotic illness, attending a special learning disability school or contained in secure care were excluded from the study.

Setting: Eight child and adolescent mental health centres, northwest England; August 2002 to August 2006.

Intervention: Manual-based developmental group psychotherapy (DGP) plus routine care versus routine care alone. Group psychotherapy was designed for self-harming adolescents and integrated techniques from cognitive-behavioural therapy, dialectical therapy and group psychotherapy. Sixweekly group therapy sessions were followed by weekly groups as long as needed. Routine care was provided according to clinical judgement by local child and adolescent mental health services and did not include any group interventions.

Outcomes: Primary outcome: frequency of episodes of selfharm over defined periods (12 months before baseline, between baseline and 6-month assessment, and between 6and 12 -month assessments). This was assessed by face-to-face structured, validated self-harm interview with the participant every 3 months. Secondary outcomes: severity of self-harm (rated based on predefined rating scheme), mood disorder (Mood and Feelings Questionnaire), suicidal ideation (Suicidal Ideation Questionnaire) and global functioning (Health of the Nation Outcome Scales for Children and Adolescents). A costeffectiveness analysis was also performed that included costs to the health, social care, education and criminal justice sectors. Unit costs were for the financial year 2005-2006.

Patient follow-up: $98.1 \%$ completed the study and were included in the analysis.

\section{METHODS}

Design: Randomised control trial.

Allocation: Concealed.

Blinding: Single blind (assessors).

Follow-up period: 12 months (treatment continued throughout this period).

\section{MAIN RESULTS}

The frequency of self-harm episodes decreased over the study period for the entire cohort. The proportion of adolescents that self-harmed weekly fell from 25.8\% (94/364) at baseline to $15.4 \%(56 / 364)$ after 6 months and to $7.7 \%(28 / 364)$ after 1 year. However, there was little evidence of additional benefit of group therapy when added to routine care. Adding group therapy did not reduce the frequency of self-harm compared with routine care alone (approximate geometric mean number of self-harm events in the first 6 months: 4.6 with group therapy vs 4.4 with routine care and that from 6 months to 1 year: 2.0 with group therapy vs 2.1 with routine care; proportional OR (POR) at 6 months: 0.99 (95\% CI 0.68 to $1.44, \mathrm{p}=0.95)$ and at 1 year: 0.88 (95\% CI 0.59 to $1.30, p=0.52))$. Secondary outcomes also improved across the whole cohort, but again with no significant difference in improvement between the groups. The total costs per adolescent in group psychotherapy were higher although the difference was not significant ( 221781 with group therapy versus $£ 15372$ with routine care alone, $\mathrm{p}=0.132$ ). The incremental cost-effectiveness of group therapy was $£ 2020$ per $1 \%$ increase in the proportion of young people not self-harming.

\section{CONCLUSIONS}

Adding DGP to routine care did not improve frequency of self-harm or other measured outcomes over 1 year among adolescents who had repeatedly self-harmed. It was not a cost-effective addition to routine care.

\section{ABSTRACTED FROM \\ Green JM, Wood AJ, Kerfoot MJ, et al. Group therapy for adolescents with repeated self harm: randomised controlled trial with economic evaluation. BMJ 2011;342:d682.}

Correspondence to: Professor Jonathan M Green, Psychiatry Research Group, University of Manchester, Manchester M13 9PL, UK; jonathan.green@manchester ac.uk

Sources of funding: Funded by The Health Foundation and sponsored by the University of Manchester.

Notes and references are available online only at http://ebmh.bmi.com
A bout $10 \%$ of adolescents will have self-harmed by the time they are 16 . Self-harm is one of the strongest predictors of suicide. Green and colleagues undertook a large and well-conducted trial of developmental group psychotherapy (DGP) versus usual care, and no clinical or financial advantages of DGP were found.

Among the possible explanations, it may be that the number of treatment sessions (about 10) was insufficient as treatment effects may take longer to develop. Adolescents, however, have a high treatment drop out rate. ${ }^{1}$ The well-conducted usual care may have reduced possible differences in efficacy of DGP. Finally, it may be that the natural history of self-harm in adolescents is better then previously thought and the result represents a regression to the mean.

There are no independently replicated studies demonstrating a reduction of self-harm repetition in adolescents published till date. Definition of self-harm compounds the problem. Many US authors research adolescents with suicidal and non-suicidal self-harm separately; many others (including Green and colleagues) research self-harm irrespectively of the suicidal intent.

Trials of cognitive-behavioural therapy (CBT), dialectic behaviour therapy (DBT), mentalisation-based therapy and family therapy are yet to report results. It is clear, however, that several wider systems (school, family and peers) play important roles in modifying adolescent self-harm, ${ }^{2}$ and future research must take these into account.

A judicial use of adult self-harm literature may be sensible in the absence of evidence-based interventions for adolescents. DBT and CBT (especially its problem solving component) tend to reduce suicidal self-harm in adults. Second-generation antipsychotics, mood stabilisers and $\omega-3$ fatty acids may be of some benefit in adults with Borderline Personality Disorder many of whom self-harm.

\section{Dennis Ougrin}

Child and Adolescent Psychiatry, King's College London, London, UK

Competing interests None. 